\title{
PERSPECTIVES
}

\section{Enhancing Scientific Foundations to Ensure Reproducibility}

\section{A New Paradigm}

Terry Hsieh, Max H. Vaickus, and Daniel G. Remick

From the Department of Pathology and Laboratory Medicine, Boston University School of Medicine, Boston, Massachusetts

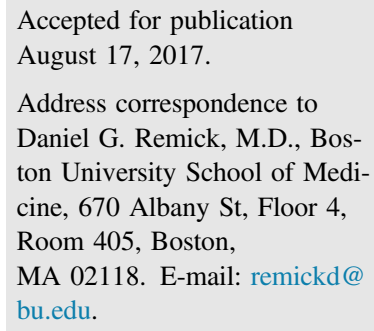

\begin{abstract}
Progress in science is dependent on a strong foundation of reliable results. The publish or perish paradigm in research, coupled with an increase in retracted articles from the peer-reviewed literature, is beginning to erode the trust of both the scientific community and the public. The NIH is combating errors by requiring investigators to follow new guidelines addressing scientific premise, experimental design, biological variables, and authentication of reagents. Herein, we discuss how implementation of NIH guidelines will help investigators proactively address pitfalls of experimental design and methods. Careful consideration of the variables contributing to reproducibility helps ensure robust results. The $\mathrm{NIH}$, investigators, and journals must collaborate to ensure that quality science is funded, explored, and published. (Am J Pathol 2018, 188: 6-10; https://doi.org/10.1016/j.ajpath.2017.08.028)
\end{abstract}

Reproducibility is fundamental to the progress of science. The publish or perish paradigm that has long permeated science pressures investigators to publish novel data in high-profile journals to secure academic advancement. Recently, the number of retractions from journals has increased significantly ${ }^{1}$ and has reached such proportions that even the mainstream media has taken notice. ${ }^{2}$ Although the Office of Research Integrity governs acts of willful misconduct, the NIH has issued several notices and assembled training modules designed to combat passive errors introduced by scientists (Clearinghouse for Training Modules to Enhance Data Reproducibility, https:// www.nigms.nih.gov/training/pages/clearinghouse-for-trainingmodules-to-enhance-data-reproducibility.aspx, last accessed August 8, 2017). Passive errors are considered as those generated unintentionally, such as those from flawed experimental design or overlooking variables. These $\mathrm{NIH}$ modules address development of rigorous experimental design, accounting for biological variables, and validation of key resources. Herein, we discuss the importance of these topics and the potential impacts on the future of biological science. Table 1 contains a synopsis of the NIH areas of focus, pitfalls present in many grant applications, and recommendations to correct these deficiencies. Since 2017, future grant applications will require investigators and reviewers to address and evaluate these areas. Implementation and adherence to these guidelines will improve the quality of science.

\section{Blinding}

Thoughtful experimental design is the first step in generating robust data. Important design aspects, such as blinding and randomization, may often be overlooked in preclinical science, even though they are considered routine in clinical trials. Although they are time-consuming, blinding and randomization guard against confirmation bias. ${ }^{3,4}$ The lack of blinding in clinical and drug development can lead to aberrant results, which could affect reproducibility. A metaanalysis of 250 controlled trials saw a larger estimate of treatment effects in the nonblinded trials compared with those

\footnotetext{
Supported by NIH grants R01GM82962 (D.G.R.), R21AI112887 (D.G.R.), R01GM97320 (D.G.R.), T32HL 007501 (D.G.R.), T32GM86308 (D.G.R.), R21AA022122 (D.G.R.), R21AI112887 (D.G.R.), T32HL 007035 (T.H.), T32007309 (T.H.), and R01GM117519 (D.G.R.).

T.H. and M.H.V. contributed equally to this work.

Disclosures: None declared.
} 
Table 1 Pitfalls and Recommendations for the NIH Areas of Focus on Rigor and Reproducibility

\begin{tabular}{|c|c|c|}
\hline NIH area of focus & Pitfalls in application & Recommendations \\
\hline Scientific premise & $\begin{array}{l}\text { Study is based on unreliable data } \\
\text { Non-hypothesis-driven study } \\
\text { Lack of supporting preliminary data }\end{array}$ & $\begin{array}{l}\text { Critically analyze the foundation of research } \\
\text { Concrete hypothesis to generate mechanistic insights } \\
\text { Stronger justifications for work if based on ideas with } \\
\text { limited literature support }\end{array}$ \\
\hline Scientific rigor & $\begin{array}{l}\text { Confirmation bias } \\
\text { Poor study design } \\
\text { Limited inclusion of methods and analyses }\end{array}$ & $\begin{array}{l}\text { Clear study design } \\
\text { Include blinding and randomization in experiments (if } \\
\text { omitted or unable, include data or justification for } \\
\text { transparency) }\end{array}$ \\
\hline Biological variables & $\begin{array}{l}\text { Omission of sex differences without justification } \\
\text { because they can influence both animal models and } \\
\text { cell lines }\end{array}$ & $\begin{array}{l}\text { Include both sexes for in vitro and in vivo studies } \\
\text { Justification for omission of sex } \\
\text { Consideration of translational issues in human disease } \\
\text { and therapy }\end{array}$ \\
\hline Authentication & $\begin{array}{l}\text { Assumption of vendor reliability/equivalence } \\
\text { Inconsistency of reagent/resource over time }\end{array}$ & $\begin{array}{l}\text { Use databases for validation (ie, http://www.cstl.nist. } \\
\text { gov/strbase or http://antibodyregistry.org) } \\
\text { Consistently check quality of both common and key } \\
\text { reagents } \\
\text { Internal validation of results } \\
\text { Clear sources of reagents and, if possible, open to peer } \\
\text { validation }\end{array}$ \\
\hline
\end{tabular}

These guidelines (https://grants.nih.gov/reproducibility/index.htm) pertain to grant applications, but the principles are broadly applicable to scientific investigation. No specific additional pages are allotted in the grant application to address these issues. It is expected that the issues will be addressed in the research plan. Blinding: withholding information about experimental group allocation from participant and/or tester to reduce confirmation bias. Randomization: use of chance methods or algorithms to allocate subjects into groups to prevent selection bias. Reproducibility: ability of experimental results and analyses to be duplicated (either by the same or an independent investigator).

trials that were blinded. ${ }^{5}$ This effect was also observed in a preclinical model of multiple sclerosis. ${ }^{6}$ It has been suggested that some nonreproducible treatment effects could stem from a lack of blinding. In one analysis, 53 articles on cancer research were repeated by an outside laboratory; only $11 \%$ were reproducible. Of the nonreproducible articles, the data were routinely analyzed without blinding, among other omissions in careful study design. ${ }^{7}$ Although all preclinical experiments may not need these steps, rigorous experimental design can aid in preventing bias. Appropriately designed preclinical studies will help to avoid the launch of expensive clinical trials based on tenuous foundations.

Lack of rigorous experimental design may have contributed to the preclinical success of NXY-059, a free radical scavenger used in models of stroke, which subsequently failed in the Stroke-Acute Ischemic NXY Treatment II (SAINT II) clinical trials. ${ }^{8}$ In a literature search, Macleod et $\mathrm{al}^{8}$ found that the treatment effect seen in nonblinded preclinical trials of NXY-059 was significantly higher than in studies that used blinding and randomization. Lack of blinding may have led to overstating the benefits of the preclinical results and, thus, a premature graduation to the more demanding clinical trials where, once blinding, randomization, and rigorous experimental methods were applied, the benefit of treatment with NXY-059 was absent. ${ }^{9}$

\section{Randomization}

Despite the importance of randomization to ensure a reproducible and rigorous experimental method, ${ }^{10}$ the practice is often neglected. In a review of the first 100 articles published in Cancer Research in 2010 that included animal experiments, only $28 \%$ reported randomization. ${ }^{11}$ Another review of animal models of various neurological disorders reported that only a third of the studies reported randomization. ${ }^{12}$ The low adherence to randomization in preclinical models is concerning because meta-analyses have shown that studies without randomization overestimate the size of the treatment effect compared with randomized studies. ${ }^{13,14}$ Hirst et al ${ }^{15}$ conducted an aggregate analysis of systematic reviews involving various animal models across a wide variety of disease processes. In their analysis of randomization, totaling 121,784 animals, randomized trials decreased effect sizes by a moderate, but statistically significant, amount. Preclinical abstracts that did not include randomization or blinding were more likely to report a difference compared with those incorporating both techniques, and abstracts that omitted both techniques were much more likely to report positive findings. ${ }^{16}$ Similar to a lack of blinding, lack of randomization may exaggerate the preclinical efficacy, resulting in a clinical trial based on less than robust data.

\section{Sex as a Biological Variable}

Sex and gender are not the same, although they are often used interchangeably. Sex refers to biological and genetic characteristics, whereas gender is the social construct of role and behavior. In line with the drive for robust study design is the requirement for critical evaluation of the biological variables that may affect the studies. A recent NIH notice 
(http://grants.nih.gov/grants/guide/notice-files/NOT-OD15-102.html, last accessed August 8, 2017) emphasized consideration of sex as a biological variable. Researchers will be required to account for sex differences in their research strategy or provide strong justification for using a single sex. Accordingly, researchers will be prompted to develop data analysis plans to stratify by sex to identify potential sex-based differences in their model of choice. To aid investigators, the Office of Research on Women's Health provides summaries of sex and gender influences on research, with the goal of increasing reproducibility and translation of study results (https://orwh.od.nih.gov/ research/sex-gender, last accessed August 8, 2017). A recent article by Miller et $\mathrm{al}^{17}$ highlights the importance of sex consideration in preclinical research and provides useful examples of proper experimental design to address these differences.

\section{Considerations of Sex}

\section{Animals}

NIH-supported clinical research contains an almost equal distribution of sex. This is, in part, because of the focus of the NIH on inclusion of women in clinical studies and the stringent regulations governing human research. Animal studies exhibit much more variability. A recent analysis of surgical research showed that of the articles that specified the sex of animals used, $80 \%$ used exclusively male animals. ${ }^{18}$ On the other hand, dermatological research exhibits a bias toward female animals. ${ }^{19}$ Preclinical research historically used primarily male animals to avoid variation from the estrous cycle in female animals. However, a metaanalysis of preclinical studies showed that female mice, regardless of estrous cycle stage, did not have significantly greater variability compared with male mice. ${ }^{20}$ The largest source of variation was actually the method of housing. Fighting in male cages caused variation, but there was a greater contribution to variability for mice housed in a group compared with by themselves, regardless of sex. ${ }^{20}$

The full range of differences between males and females is beyond the scope of this article, but incorporation of this issue in translational research is needed. Preclinical sexual dimorphisms can provide mechanistic insights, as seen in a model of pneumococcal pneumonia, where female C57BL/6 mice exhibited improved survival and reduced proinflammatory cytokine profiles compared with male mice. ${ }^{21}$ Yang et $\mathrm{al}^{22}$ reproduced this survival difference and also showed that alveolar macrophages from female mice had increased bacterial killing and that this dimorphism existed in human alveolar macrophages. This effect is enhanced by estrogen-mediated activation of nitric oxide synthase- 3 and can be mimicked by statin treatment. Armed with these preclinical data, their retrospective analysis showed that statins, estrogen, or both, in a female cohort, significantly reduced the risk of pneumonia requiring hospitalization.
Adherence to this initiative should help to reduce variation, improve reproducibility, and potentially lead to clinically relevant findings.

\section{Cell Culture}

Even preclinical studies using cell culture should not neglect possible sexual dimorphisms. For many experiments, it might seem a trivial issue to control for the gender of isolated cells. However, investigators may control for genetic deletions or additions in their studies, but may ignore the presence of the $\mathrm{Y}$ chromosome or inactivated second $\mathrm{X}$ chromosome, which fundamentally differ from each other. Shah et $\mathrm{al}^{23}$ discussed the importance of sex identification in cell culture studies. They highlighted several studies in which sexual dimorphism, potentially ranging between $14 \%$ and $70 \%$ in various tissues, could help to elucidate physiological mechanisms. Without proper consideration, these sex differences represent a clandestine source of variability. An emerging cellular source of therapy is induced pluripotent stem cells (iPSCs). Given the mechanism of generating iPSCs, we might not consider sexual dimorphism important. However, Anguera et $\mathrm{al}^{24}$ demonstrated that significant differences exist between male and female human iPSCs. Using female-specific markers, they differentiated three classes of female iPSCs. One subset displayed significant oncogenic potential, including up-regulation of X-linked oncogenes, enhanced growth rate in vitro, and reduced ability to differentiate in vivo. Inconsistency in X-chromosome inactivation resulted in epigenetic variability of female iPSCs that is not found in male iPSCs. Thus, use of female iPSCs in therapy may warrant a caveat not found in male iPSCs. ${ }^{24}$ To meet the NIH guidelines, studies using primary cells should include sex reporting and analysis, similar to those used in animal studies.

Proponents of this policy argue that, especially in preclinical models, consideration of sexual dimorphism is important for clinical translation. ${ }^{25}$ This issue is not without controversy, because opponents argue that these new NIH policies push documentation, not experimentation, and will not translate to equalizing health disparities between men and women. ${ }^{26}$ It must be acknowledged that these policies might divert resources from an investigator's primary focus and that mounting costs are a barrier to adoption of this policy. However, accounting for sexual dimorphisms will at the least strengthen data, if not lead to identification of unique mechanisms or novel findings.

\section{Validation of Key Resources}

The final piece of these new proposals is the validation of key reagents in research. Animals, antibodies, cell lines, and chemicals critical to the outcome of experiments should be routinely vetted during their use. Recent recommendations from the Federation of American Societies for Experimental Biology, 
generated from the 2015 Science Policy Symposium, provided practical strategies that encourage reproducibility regarding resources and are a useful reference to those in the research world (https://www.faseb.org/Portals/2/PDFs/opa/2016/ FASEB_Enhancing\%20Research\%20Reproducibility.pdf, last accessed August 8, 2017). The consistency of key reagents is necessary for the reproducibility of published work. Over passages or generations, cell lines or animal strains may diverge from the initial progenitor. Human cell lines can be identified by short tandem repeat loci and by comparison against a database, such as that provided by ATCC (Manassas, VA). However, for newly characterized cell lines or ones from other species, investigators should develop and include signature sequences to provide validation. A frequently updated database similar to STRBase for human short tandem repeat identification (http://www.cstl.nist.gov/ strbase, last accessed August 8,2017) will be helpful for the research community to share identifying markers for their cell lines.

Antibodies, chemicals, and media are important factors reported in studies. Variations in the sources or formulation of these key reagents may affect reproducibility. A study attempting to identify reported resources found that across several journals, $54 \%$ of resources were not uniquely identifiable. ${ }^{27}$ The reproducibility of research suffers if key reagents are not properly identified or reported. Antibodies are in ubiquitous use, but can exhibit extensive variability over time or between suppliers. The Antibody Registry (http:// antibodyregistry.org, last accessed August 8, 2017) is a website that catalogs antibody information from peers and suppliers, providing a resource for investigators to identify a cited antibody. However, internal validation should still be performed to verify the specificity of the reagents. ${ }^{28}$ Reagent consistency should be tested periodically to ensure reproducibility of one's own work as well as the work of others. Grant reviewers, journal editors, and researchers should all be cognizant of these factors and strive for the enumeration of key resources to ensure reproducible research findings.

\section{Conclusion}

Although the implementation of these guidelines may meet resistance because of the added cost and effort required by an already overburdened laboratory, they are important measures to ensure reproducibility and maintain trust in science. In addition to the NIH, journals and investigators must collaborate to further the mission of publishing reproducible results. Numerous scientific associations and journal editors, including The American Journal of Pathology, endorsed principles and guidelines to promote rigorous science (Principles and Guidelines for Reporting Preclinical Research, https://www.nih.gov/research-training/rigor-reproducibility/ principles-guidelines-reporting-preclinical-research, last accessed August 8, 2017). As scientists, we build on the work of our contemporaries and predecessors. Efforts by all members of the scientific community will produce a solid foundation of publications for confident future progress. Furthermore, implementation of these policies will help to train future generations to account for these variables and continue rigorous investigation. Despite the pervasive drive to publish, we must strive to maintain the rigor and reproducibility of our work. Irreproducible and retracted results diminish public trust in science, which fuels proponents of slashing NIH budgets that ultimately perpetuates this paradigm. We hope that embracing these policies will lead to quality, reproducible work that not only restores public trust, but restores the ideal that published science is proper science.

\section{References}

1. Fanelli D: Why growing retractions are (mostly) a good sign. PLoS Med 2013, 10:e1001563

2. Carey B: Science, now under scrutiny itself. The New York Times, June 15, 2015. pp. D1

3. Kilkenny C, Parsons N, Kadyszewski E, Festing MF, Cuthill IC, Fry D, Hutton J, Altman DG: Survey of the quality of experimental design, statistical analysis and reporting of research using animals. PLoS One 2009, 4:e7824

4. Kilkenny C, Browne WJ, Cuthill IC, Emerson M, Altman DG: Improving bioscience research reporting: the ARRIVE guidelines for reporting animal research. PLoS Biol 2010, 8:e1000412

5. Schulz KF, Chalmers I, Hayes RJ, Altman DG: Empirical evidence of bias: dimensions of methodological quality associated with estimates of treatment effects in controlled trials. JAMA 1995, 273:408-412

6. Vesterinen HM, Sena ES, ffrench-Constant C, Williams A, Chandran S, Macleod MR: Improving the translational hit of experimental treatments in multiple sclerosis. Mult Scler 2010, 16:1044-1055

7. Begley CG, Ellis LM: Drug development: raise standards for preclinical cancer research. Nature 2012, 483:531-533

8. Macleod MR, van der Worp HB, Sena ES, Howells DW, Dirnagl U, Donnan GA: Evidence for the efficacy of NXY-059 in experimental focal cerebral ischaemia is confounded by study quality. Stroke 2008, 39:2824-2829

9. Shuaib A, Lees KR, Lyden P, Grotta J, Davalos A, Davis SM, Diener HC, Ashwood T, Wasiewski WW, Emeribe U: NXY-059 for the treatment of acute ischemic stroke. N Engl J Med 2007, 357:562-571

10. Festing MF, Altman DG: Guidelines for the design and statistical analysis of experiments using laboratory animals. ILAR J 2002, 43:244-258

11. Hess KR: Statistical design considerations in animal studies published recently in cancer research. Cancer Res 2011, 71:625

12. van der Worp HB, Howells DW, Sena ES, Porritt MJ, Rewell S, O'Collins V, Macleod MR: Can animal models of disease reliably inform human studies? PLoS Med 2010, 7:e1000245

13. Sena E, van der Worp HB, Howells D, Macleod M: How can we improve the pre-clinical development of drugs for stroke? Trends Neurosci 2007, 30:433-439

14. Macleod MR, O'Collins T, Horky LL, Howells DW, Donnan GA: Systematic review and metaanalysis of the efficacy of FK506 in experimental stroke. J Cereb Blood Flow Metab 2005, 25:713-721

15. Hirst JA, Howick J, Aronson JK, Roberts N, Perera R, Koshiaris C, Heneghan $\mathrm{C}$ : The need for randomization in animal trials: an overview of systematic reviews. PLoS One 2014, 9:e98856

16. Bebarta V, Luyten D, Heard K: Emergency medicine animal research: does use of randomization and blinding affect the results? Acad Emerg Med 2003, 10:684-687

17. Miller LR, Marks C, Becker JB, Hurn PD, Chen WJ, Woodruff T, McCarthy MM, Sohrabji F, Schiebinger L, Wetherington CL, 
Makris S, Arnold AP, Einstein G, Miller VM, Sandberg K, Maier S, Cornelison TL, Clayton JA: Considering sex as a biological variable in preclinical research. FASEB J 2016, 31:29-34

18. Yoon DY, Mansukhani NA, Stubbs VC, Helenowski IB, Woodruff TK, Kibbe MR: Sex bias exists in basic science and translational surgical research. Surgery 2014, 156:508-516

19. Kong BY, Haugh IM, Schlosser BJ, Getsios S, Paller AS: Mind the gap: sex bias in basic skin research. J Invest Dermatol 2016, 136:12-14

20. Prendergast BJ, Onishi KG, Zucker I: Female mice liberated for inclusion in neuroscience and biomedical research. Neurosci Biobehav Rev 2014, 40:1-5

21. Kadioglu A, Cuppone AM, Trappetti C, List T, Spreafico A, Pozzi G, Andrew PW, Oggioni MR: Sex-based differences in susceptibility to respiratory and systemic pneumococcal disease in mice. J Infect Dis 2011, 204:1971-1979

22. Yang Z, Huang YC, Koziel H, de Crom R, Ruetten H, Wohlfart P, Thomsen RW, Kahlert JA, Sorensen HT, Jozefowski S, Colby A, Kobzik L: Female resistance to pneumonia identifies lung macrophage nitric oxide synthase-3 as a therapeutic target. Elife 2014, 3

23. Shah K, McCormack CE, Bradbury NA: Do you know the sex of your cells? Am J Physiol Cell Physiol 2014, 306:C3-C18
24. Anguera MC, Sadreyev R, Zhang Z, Szanto A, Payer B, Sheridan SD, Kwok S, Haggarty SJ, Sur M, Alvarez J, Gimelbrant A, Mitalipova M, Kirby JE, Lee JT: Molecular signatures of human induced pluripotent stem cells highlight sex differences and cancer genes. Cell Stem Cell 2012, 11:75-90

25. Klein SL, Schiebinger L, Stefanick ML, Cahill L, Danska J, de Vries GJ, Kibbe MR, McCarthy MM, Mogil JS, Woodruff TK, Zucker I: Opinion: sex inclusion in basic research drives discovery. Proc Natl Acad Sci U S A 2015, 112:5257-5258

26. Richardson SS, Reiches M, Shattuck-Heidorn H, LaBonte ML, Consoli T: Opinion: focus on preclinical sex differences will not address women's and men's health disparities. Proc Natl Acad Sci U S A 2015, 112:13419-13420

27. Vasilevsky NA, Brush MH, Paddock H, Ponting L, Tripathy SJ, Larocca GM, Haendel MA: On the reproducibility of science: unique identification of research resources in the biomedical literature. PeerJ 2013, 1:e148

28. Bordeaux J, Welsh A, Agarwal S, Killiam E, Baquero M, Hanna J, Anagnostou V, Rimm D: Antibody validation. Biotechniques 2010, 48:197-209 